\title{
Editorial
}

Theme: Formulation and Delivery of Macromolecules

Guest Editors: Jason T. McConville and Javier O. Morales

\section{Formulation and Delivery of Macromolecules}

\author{
Javier O. Morales ${ }^{1,2,3}$
}

Received 1 December 2016; accepted 2 December 2016; published online 9 December 2016

The field of macromolecule drug development (also known in the literature as biologics, biomacromolecules, and biotechnology drugs) has sharply grown over the past 15 years (1). With a steady growth in total sales, sales per drug type (i.e., therapeutic proteins and peptides, monoclonal antibodies, or vaccines), and innovation, macromolecule drug development continues to make a strong case in contrast with small-molecule drugs $(2,3)$. While most marketed products and clinical trials on macromolecule drugs are injectable dosage forms, there is a drive for more convenient dosage forms evidenced in the body of scientific literature, numerous clinical trials, and a handful of successful products for alternative routes of administration.

While extravascular administration has been widely investigated and has shown potential, there are many difficulties associated with absorption of therapeutic macromolecules into the body. For example, oral delivery of macromolecules for intestinal absorption faces a number of well-known limitations including instability in gastric $\mathrm{pH}$, proteolytic enzyme content in the upper gastrointestinal (GI) tract, and insufficient permeation and bioavailability; therefore, encapsulation, targeted release, and novel permeation enhancers have been a focus area of research (4). With the difficulties of GI tract absorption, alternative routes of delivery have also been investigated, including buccal/sublingual, transdermal, and nasal/pulmonary. This special theme of AAPS PharmSciTech explores specific aspects in the state of the art in macromolecule delivery and showcases innovative research strategies

\footnotetext{
${ }^{1}$ Department of Pharmaceutical Science and Technology, School of Chemical and Pharmaceutical Sciences, University of Chile, Santos Dumont 964, 4to piso, Of\# 09, Independencia, Santiago 8380494, Chile.

${ }^{2}$ Advanced Center for Chronic Diseases (ACCDiS), Santiago, 8380494, Chile.

${ }^{3}$ To whom correspondence should be addressed. (e-mail: jomorales@ciq.uchile.cl)
}

intended to improve absorption and performance of dosage forms containing macromolecular drugs.

In our review in this Themed Edition, Montenegro-Nicolini and Morales describe the major advancements in mucoadhesive films as dosage forms for buccal administration of macromolecule drugs (5). The review presents the stratified mucosal epithelium as an absorption barrier and introduces strategies to enhance macromolecule permeation. The authors identify solvent casting as the conventional choice for film development; however, recent advancements in hot melt extrusion (6) and inkjet printing (7) could provide new avenues for buccal film product development.

Hot melt extrusion of solid dosage forms for macromolecule drug development has been avoided in the past due to potential drug degradation due to the high temperatures and shear achieved during the process. Nonetheless, efforts in using low-glass transition temperature polymers and optimized processing conditions have shown promise. In their article, Cossé et al. focus on systematically investigating all relevant extrusion variables as well as using a lowtemperature processing polymer (6). After suitable formulation development, the authors showed that the extruded implants were able to sustain bovine serum albumin (BSA) release for weeks while maintaining very limited conformation changes in the BSA structure.

In an alternative approach for macromolecule drug formulation in nanostructured systems, two articles detail the development and characterization of niosomes as delivery systems $(8,9)$. Firstly, a formulation optimization study was conducted and BSA release properties of niosomes determined. This investigation additionally showed a cost-effective method for BSA quantification in niosomes and similar vesicles (8). Secondly, similar span 60 and cholesterol niosomes were loaded with basic fibroblast growth factor, incorporated in agarose hydrogel as a scaffold, and evaluated for their in vitro cell proliferation performance (9). The said delivery system was successful in controlling drug release and improving cell proliferation even in comparison with the niosomal suspension. 
As most macromolecule drug products are injectables, a thorough depiction of structural stability during storage is required during development. In their article, Zheng et al. investigate the influence of $\mathrm{pH}$ and buffer species in an IgG 1 subtype therapeutic monoclonal antibody A (mAbA) (10). Through a combination of dynamic light scattering, differential scanning calorimetry, size-exclusion chromatography, hydrophobic interaction chromatography, gel electrophoresis, and liquid chromatography mass spectrometry as characterization tools, the authors systematically studied the antibody degradation pathways. While this research highlights the need for specificity in monoclonal antibody characterization of degradation, the design used and techniques employed could be useful for investigating similar drugs.

Finally, an approach to study supramolecular inclusion complexes using lab techniques and in silico calculations is included in this Themed Edition and could be used in macromolecule drug development (11). dos Passos Menezes et al. investigated the physicochemical properties of inclusion complexes through various spectroscopic techniques and used a molecular modeling approach to contrast their results and show similar tendencies.

The continued growth in the macromolecule drug field will move forward new entities for formulation and thus push further the drive for development of alternative dosage forms. While much is known in formulating and developing injectables, only few successful medicines for alternative routes have been approved and are marketed. Research and innovation is expected to continue bringing new molecules to formulate, and the research in alternative routes of administration is likely to result in more availability of macromolecule drug products.

\section{REFERENCES}

1. Pagels RF, Prud'homme RK. Polymeric nanoparticles and microparticles for the delivery of peptides, biologics, and soluble therapeutics. J Control Release. 2015;219:519-35.

2. Bain B, Shortmoor J. Pharma market trends 2010. Pharm Technol. 2010.

3. Miller KL, Lanthier M. Regulatory watch: innovation in biologic new molecular entities: 1986-2014. Nat Rev Drug Discov. 2015;14:83.

4. Brayden DJ, Alonso M-J. Oral delivery of peptides: opportunities and issues for translation. Adv Drug Deliv Rev. 2016;106(Part B):193-5.

5. Montenegro-Nicolini M, Morales JO. Overview and future potential of buccal mucoadhesive films as drug delivery systems for biologics. AAPS PharmSciTech. 2016. doi:10.1208/s12249-016-0525-z.

6. Cossé A, König C, Lamprecht A, Wagner KG. Hot melt extrusion for sustained protein release: matrix erosion and in vitro release of PLGA-based implants. AAPS PharmSciTech. 2016. doi:10.1208/s12249-016-0548-5.

7. Montenegro-Nicolini M, Miranda V, Morales JO. Inkjet printing of proteins: an experimental approach. AAPS J In Press. 2016:1-10.

8. Moghassemi S, Hadjizadeh A, Omidfar K. Formulation and characterization of bovine serum albumin-loaded niosome. AAPS PharmSciTech. 2016. doi:10.1208/s12249-016-0487-1.

9. Moghassemi S, Hadjizadeh A, Hakamivala A, Omidfar K. Growth factor-loaded nano-niosomal gel formulation and characterization. AAPS PharmSciTech. 2016. doi:10.1208/ s12249-016-0579-y.

10. Zheng S, Qiu D, Adams M, Li J, Mantri RV, Gandhi R. Investigating the degradation behaviors of a therapeutic monoclonal antibody associated with $\mathrm{pH}$ and buffer species. AAPS PharmSciTech. 2015. doi:10.1208/s12249-015-0403-0.

11. dos Passos Menezes P, dos Santos PBP, Dória GAA, et al. Molecular modeling and physicochemical properties of supramolecular complexes of limonene with $\alpha$ - and $\beta$-cyclodextrins. AAPS PharmSciTech. 2016. doi:10.1208/s12249-016-0516-0. 